Rückert-John, Jana

\title{
Der Wandel des Ernährungsalltags als Herausforderung für die
} Ernährungs- und Verbraucherkommunikation

Haushalt in Bildung \& Forschung 4 (2015) 3, S. 39-50

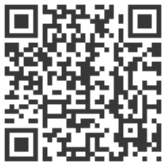

Quellenangabe/ Reference:

Rückert-John, Jana: Der Wandel des Ernährungsalltags als Herausforderung für die Ernährungsund Verbraucherkommunikation - In: Haushalt in Bildung \& Forschung 4 (2015) 3, S. 39-50 - URN: urn:nbn:de:0111-pedocs-203800 - DOI: 10.25656/01:20380

https://nbn-resolving.org/urn:nbn:de:0111-pedocs-203800

https://doi.org/10.25656/01:20380

in Kooperation mit / in cooperation with:

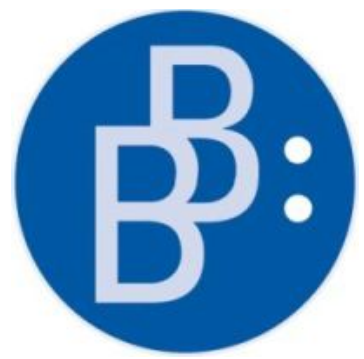

https://www.budrich.de

\section{Nutzungsbedingungen}

Gewährt wird ein nicht exklusives, nicht übertragbares, persönliches und beschränktes Recht auf Nutzung dieses Dokuments. Dieses Dokument ist ausschließlich für den persönlichen, nicht-kommerziellen Gebrauch bestimmt. Die Nutzung stellt keine Übertragung des Eigentumsrechts an diesem Dokument dar und gilt vorbehaltlich der folgenden Einschränkungen: Auf sämtlichen Kopien dieses Dokuments müssen alle Urheberrechtshinweise und sonstigen Hinweise auf gesetzlichen Schutz beibehalten werden. Sie dürfen dieses Dokument nicht in irgendeiner Weise abändern, noch dürfen Sie dieses Dokument für öffentliche oder kommerzielle Zwecke vervielfältigen, öffentlich ausstellen, aufführen, vertreiben oder anderweitig nutzen. Mit der Verwendung dieses Dokuments erkennen Sie die

\section{Terms of use}

We grant a non-exclusive, non-transferable, individual and limited right to using this document

This document is solely intended for your personal, non-commercial use. Use of this document does not include any transfer of property rights and it is conditional to the following limitations: All of the copies of this documents must retain all copyright information and other information regarding legal protection. You are not allowed to alter this document in any way, to copy it for public or commercial purposes, to exhibit the document in public, to perform, distribute or otherwise use the document in public.

By using this particular document, you accept the above-stated conditions of use.

\section{Kontakt / Contact:}

\section{peDOcs}

DIPF | Leibniz-Institut für Bildungsforschung und Bildungsinformation Informationszentrum (IZ) Bildung

E-Mail:pedocs@dipf.de

Internet: www.pedocs.de

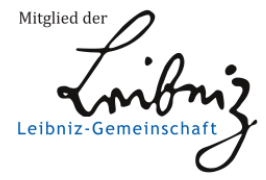




\section{Haushalt in}

Forschung

Sozioökonomische Allgemein-, Ernährungs- und Verbraucherbildung
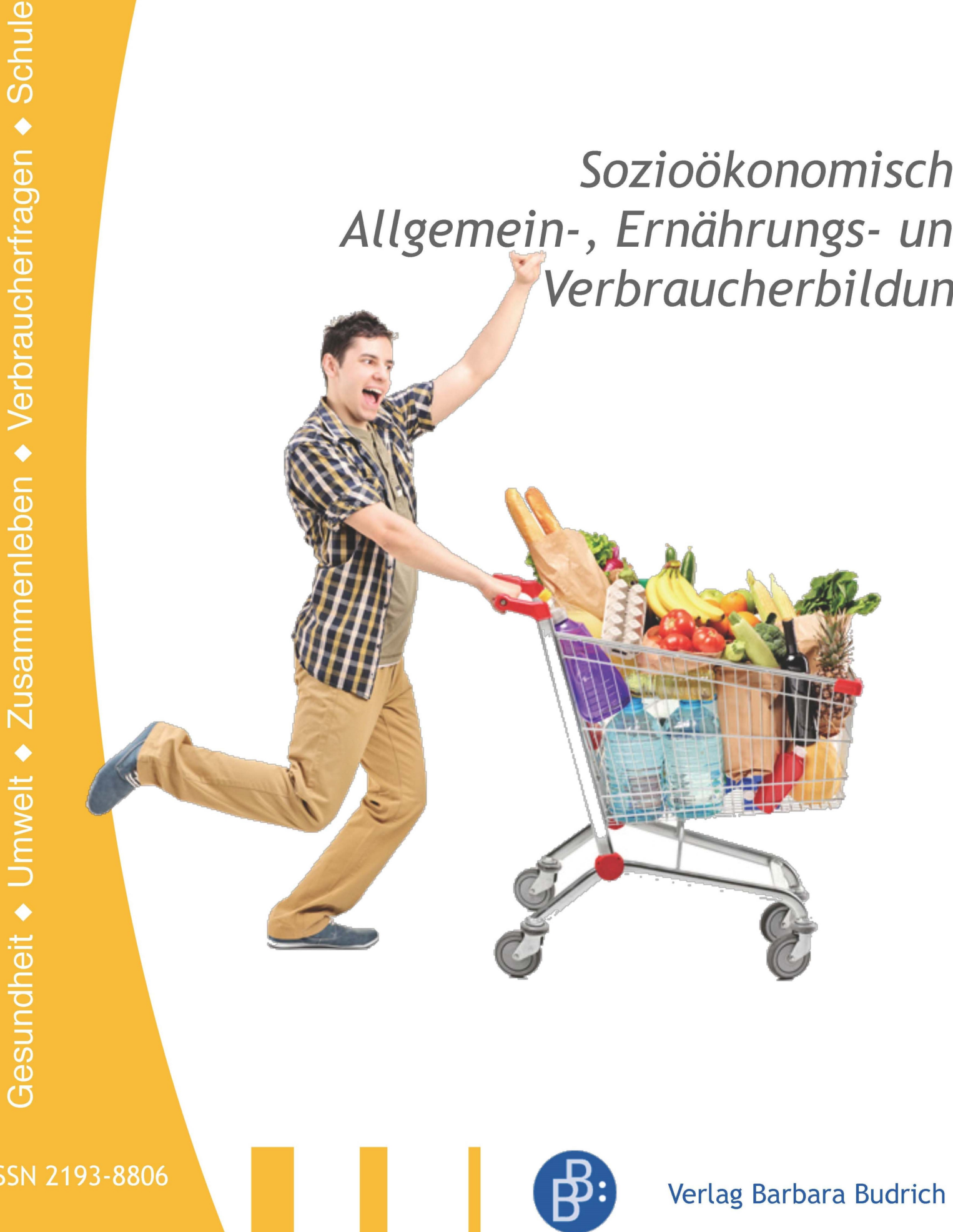
Inhaltsverzeichnis |

Werner Brandl

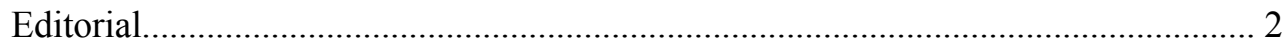

Reinhold Hedtke

Sozioökonomische Bildung................................................................................. 3

Angela Häußler

Fokus Haushalt - Überlegungen zu einer sozio-ökonomischen Fundierung der

Verbraucherbildung.

Uta Meier-Gräwe

Caring. Cooking. Cleaning. Warum wir die Arbeit des Alltags gesellschaftlich neu und geschlechtergerecht verteilen müssen.

Jana Rückert-John

Der Wandel des Ernährungsalltags als Herausforderung für die Ernährungs- und

Verbraucherkommunikation

Carolin Kölzer \& Volker Schwier

Sozioökonomische Bildung im Sachunterricht der Grundschule - mehr

als ,work, earn, play“.

Stefanie Nolte

Mit reflektierten Konsumentscheidungen die Zukunft fairändern - eine

Zukunftswerkstatt.

Uwe Becker

Alter, Armut, Schulden. Warum Prävention ein Euphemismus ist.

Werner Brandl

Reinhold Hedtke (Hrsg.). Was ist und wozu Sozioökonomie?.

Angela Häußler \& Barbara Methfessel

Uta Meier-Gräwe (Hrsg.). Die Arbeit des Alltags. Gesellschaftliche Organisation

und Umverteilung.

Anna Maria Hoff

Jana Rückert-John (Hrsg.). Soziale Innovation und Nachhaltigkeit.

Perspektiven sozialen Wandels.

HaBiFo-Fachtagung und Preis 2016. 
Jana Rückert-John

\section{Der Wandel des Ernährungsalltags als Herausforderung für die Ernährungs- und Verbraucherkommunikation}

Der Ernährungswohlstand ruft massive gesundheitliche und ökologische Probleme hervor, die einen Wandel der Ernährungspraktiken notwendig erscheinen lassen. Statt wie in bisheriger Weise eine Defizitperspektive an die alltäglichen Ernährungspraktiken anzulegen, geht es vor dem Hintergrund praxistheoretischer Ansätze darum, auf Potenzialerkennung umzustellen, um so realistische Chancen eines Wandels der Ernährungspraktiken zu bestimmen.

Schlüsselwörter: Alltag, Ernährungspraktiken, sozialer Wandel, Innovation, Kommunikation

\section{Einleitung}

Ernährungsstile, die für westliche Industrieländer als typisch angesehen werden, erachten Experten in mehrfacher Hinsicht als problematisch. Der Überfluss ruft massive gesundheitliche und ökologische Probleme hervor, die einen Wandel der Essund Ernährungspraktiken notwendig erscheinen lassen. In der Ernährungs- und Verbraucherkommunikation wird jedoch allzu häufig allein von einem mangelnden Bewusstsein und mangelndem Wissen der Konsumenten ausgegangen. Deshalb wird der individuelle Verbraucher angesprochen, sein Verhalten zu verändert. Diese Defizitperspektive aber trägt kaum bis gar keine Früchte. Eine alternative Perspektive, die an Überlegungen der soziologischen Praxistheorien anschließt, soll darum im Folgenden mit Blick auf die Ernährungs- und Verbraucherkommunikation vorgestellt werden. Es wird argumentiert, dass es darum gehen muss, den auf Praktiken gründenden Ess- und Ernährungsalltag zunächst zu verstehen und dabei die vorherrschenden Alltagslogiken anzuerkennen. Erst so informiert, sind realistische Chancen eines Wandels der Ernährungspraktiken zu bestimmen. Mit diesem alternativen Ansatz wird eine grundsätzliche Umstellung der Forschungsperspektive vorgenommen, bei der nicht - wie in bisheriger Weise - eine Defizitperspektive an die Ess- und Ernährungspraktiken angelegt, sondern auf Potenzialerkennung umgestellt wird. 


\section{Ernährungs- und Verbraucherkommunikation}

\section{Gesundheitliche und ökologische Probleme westlicher Ernährungsstile}

In westlichen Industrieländern ist heute nicht nur die ausreichende mengenmäßige Verfügbarkeit an Nahrungsmitteln für die überwiegende Mehrheit der Bevölkerung als Problem gelöst. Ebenso ist es Normalität, dass räumliche und zeitliche Restriktionen des Essens außer Kraft gesetzt sind. Dazu gehört auch, dass in urbanen Zentren der westlichen Welt nahezu rund um die Uhr gegessen werden kann. Wurde Ernährung bis in die 1960er Jahre in Deutschland und anderen westeuropäischen Ländern noch vorrangig unter dem Fokus von Nahrungssicherung diskutiert, so hat die Thematisierung von Ernährung in den letzten Dekaden eine deutliche Veränderung erfahren (Hirschfelder, 2005).

Im westlichen Kontext geht es heute weniger um die Grundsicherung der Ernährung der Bevölkerung. Vielmehr werden die Folgen der industrialisierten Erzeugung von Nahrungsmitteln mit Primat auf Wettbewerbsfähigkeit im internationalen Agrarmarkt diskutiert, die anstelle des Mangels ein Überangebot von Nahrung schaffen. Hiermit sind einerseits Fragen des Umgangs mit Nahrungswohlstand und Verschwendung bis hin zu Problemen der sogenannten Fehlernährung durch Überernährung berührt und andererseits gravierende ökologische Folgewirkungen westlicher Ernährungsstile angesprochen (Eberle et al., 2006). Dieser gesellschaftliche Wandel vom Mangel zum Überfluss stellt historisch gesehen eine einmalige Situation in Deutschland dar. Er findet unter anderem auch seinen Ausdruck in durchschnittlich sinkenden Lebensmittelausgaben der deutschen Privathaushalte. Betrug der Anteil der Ausgaben privater Haushalte für Nahrungsmittel im Jahre 1950 noch 44 Prozent - also fast die Hälfte des Haushaltseinkommens - , so nahm er über die letzten Jahrzehnte kontinuierlich ab und betrug in 2014 nur noch 13 Prozent (Statistisches Bundesamt, 2015). Diese Entwicklung wird häufig als Anzeiger für Wohlstand diskutiert, da so über die letzten Dekaden ein wachsender Anteil finanzieller Mittel für andere Konsumentscheidungen der Privathaushalte frei wurde. Als Problem erscheint diese Entwicklung aber schon, wenn bemerkt wird, dass sinkende Lebensmittelausgaben heute mit geringer Wertschätzung oder auch Lebensmittelverschwendung einhergehen. Dies könnte leicht als Zeichen für eine einseitige Wohlstandsdebatte missverstanden werden, wenn nicht offensichtlich wäre, dass Mangel und Hunger trotz Wohlstand auch in der Überflussgesellschaft anzutreffen sind (Pfeiffer, 2014). Armut drückt sich in Ländern wie Deutschland nicht einfach als Hunger aus, sondern häufig als Übergewicht und Adipositas (Max Rubner Institut, 2008).

Die gesellschaftliche Herausforderung des „Überlebens im Überfluss“ fokussiert nicht nur auf die gesundheitlich relevanten Ernährungsprobleme der sogenannten Fehlernährung, sondern gleichermaßen auf die ökologischen Folgeprobleme entlang der gesamten Wertschöpfungskette von Nahrungsmitteln: der Produktion, der Verar- 


\section{Ernährungs- und Verbraucherkommunikation}

beitung, des Transports, der Zubereitung und des Verzehrs sowie des Wegwerfens. So lassen sich vor allem am Fleischkonsum verschiedene ökologische Problemlagen aufzeigen, wie der Flächen- und Wasserverbrauch, die hiermit verbundene Wasserverunreinigung, der einhergehende Biodiversitätsverlust und die enorme Klimabelastung (Rückert-John, 2015). Hinzu kommen Probleme aus Sicht des Tierschutzes. Vor diesem Hintergrund kam der Wissenschaftliche Beirat des Bundesministeriums für Ernährung und Landwirtschaft (2015) in seinem jüngsten Gutachten zu der Einschätzung, dass die derzeitigen Haltungsbedingungen eines Großteils der Nutztiere in Deutschland nicht zukunftsfähig seien.

\section{Häufiger Lösungsansatz: Die Konsumenten sollen es richten!}

Vor dem skizzierten Problemkontext ist nun nach möglichen Lösungsansätzen und der Rolle verschiedener Akteure zu fragen. Häufig rückt mit den als problematisch erachteten Ernährungspraktiken der Konsument in den Mittelpunkt der Betrachtung. In den Problembeschreibungen der Politik, Ernährungswissenschaften und der Ernährungsberatung werden die Zunahme ernährungsbedingter Erkrankungen, wie Adipositas und Übergewicht, und ein mangelndes nachhaltiges Alltagshandeln konstatiert und auf mangelhaftes Ernährungswissen und -bewusstsein zurückgeführt. Dem individuellen Ernährungsverhalten wird damit ein grundlegendes Defizit unterstellt, wenn von fehlender Vernunft und fehlender Verantwortung die Rede ist (Rückert-John, 2014). Es wird davon ausgegangen, dass durch eine gesündere Ernährung und einem verantwortungsvolleren Konsumentenverhalten eine nachhaltigere Ernährung erreicht werden kann. Es wird erwartet, dass der Verbraucher eine „Politik mit dem Einkaufskorb" betreibt (Busse, 2006).

Vielfach werden daraufhin Interventionsmaßnahmen und Bildungsprogramme mit der Absicht initiiert, das Ernährungsverhalten unter der Maßgabe einer gesunden und nachhaltigen Ernährung zu verbessern. Auf diese Weise soll nicht zuletzt ein Beitrag zur Krankheitsprävention im Bereich der Ernährung geleistet werden. Denn ganz überwiegend werden die Ernährungspraktiken der deutschen Bevölkerung unter den normativen Prämissen der Gesundheit und Nachhaltigkeit als defizitär und mangelhaft bewertet. Einen wichtigen Stellenwert wird hierbei der Ernährungs- und Verbraucherbildung sowie der Wissensvermittlung beigemessen. Die grundlegende Annahme lautet, dass mehr Informationen und besseres Wissen zu einer bewussteren und gesünderen Ernährung führen.

Jedoch sind - trotz vieler Bemühungen - die Erfolge derartiger Interventionsund Bildungsmaßnahmen sehr begrenzt. Eher wird ein Scheitern der Ernährungsberatung konstatiert (Klotter, 2014; Reisch \& Gwozdz, 2011). Das lässt sich ebenso für Strategien und Maßnahmen im Bereich des nachhaltigen Konsums feststellen. Seit mehr als zwei Jahrzehnten werden vielfältige Nachhaltigkeitsstrategien erprobt, die 


\section{Ernährungs- und Verbraucherkommunikation}

Bürgerinnen und Bürger dazu motivieren sollen, sozial- und umweltverträglicher zu konsumieren. Vorherrschend sind auch hier Versuche, die Motive und das Wissen von Konsumentinnen und Konsumenten mittels Informationen und zum Beispiel finanzieller Anreize zu beeinflussen. Trotz einiger Teilerfolge konnte bislang keine umfassende Trendwende in Richtung Nachhaltigkeit herbeigeführt werden (Brand, 2011).

Die Ursachen für den mangelnden Erfolg derartiger Strategien und Maßnahmen sind vielfältig, sie sollen deshalb anhand einer zentralen These diskutiert werden. Gründe für das Scheitern sind vor allem darin ausmachen, dass mit dieser Problemperspektive die alltäglichen Ernährungspraktiken der Konsumentinnen und Konsumenten sowohl überschätzt als auch unterschätzt werden. Überschätzt werden die Praktiken, wenn das Wissen über gesundheitliche und ökologische Folgen der Ernährung als ausreichende Motivation für entsprechende gesundheits- und nachhaltigkeitsorientierte Ernährungspraktiken angenommen wird. Diesen Annahmen liegt das theoretische Modell des Homo oeconomicus zugrunde, bei dem das menschliche Handeln rational und nutzenmaximierend erklärt wird. Zahlreiche Studien im Bereich der Ernährungs- und auch Nachhaltigkeitsforschung haben jedoch gezeigt, dass Wissen lediglich ein Faktor bei der Gestaltung von Alltagspraktiken wie etwa der Ernährung darstellt, insbesondere wenn es um Entscheidungen für andersartige, alternative Praktiken geht. Stattdessen ist ein als ungesund oder nicht-nachhaltig bewertetes Handeln trotz eines häufig vorhandenen Wissens über jeweilige Effekte zu beobachten (z. B. BMUB/UBA 2013, John et al. ,2014).

Unterschätzt werden die Ernährungspraktiken dann, wenn allein vor dem Hintergrund abstrakter und damit alltagsferner Ernährungsempfehlungen (wie beispielsweise der DGE) die Rationalität und damit die Vernunft der jeweiligen Ernährungspraktiken angezweifelt werden. Diese Unterschätzung kommt vor allem darin zum Ausdruck, wenn die Ernährungspraktiken durch die Gegenüberstellung mit den Empfehlungen als defizitär und mangelhaft erscheinen, ohne dass dabei die tatsächlichen alltagspraktischen Umstände überhaupt in Erwägung gezogen werden. Darüber hinaus wird dem Ernährungsalltag bestimmter, nämlich bildungsferner und einkommensschwacher Zielgruppen damit überhaupt auch eine Ordnung und Eigenlogik abgesprochen. Die Ernährungspraktiken erscheinen eher wenig rational, zuweilen widersprüchlich und unlogisch. Entgegen solchen pauschalisierenden Bewertungen käme es jedoch erst einmal darauf an, die in diesem offensichtlich fremden Ernährungsalltag waltende Rationalität und Ordnung zu erkennen, um sie zu verändern.

Häufige Probleme derartiger verhaltenspräventiver Konzepte und Ansätze bestehen darin, dass mittels moralischer Kommunikation argumentieren, das heißt, dass hiermit Normen und Wertvorstellungen einer gesunden und nachhaltigen Ernährung vornehmlich einer bürgerlichen Mittelschicht vermittelt werden, die auch immer ein Urteil darüber abgeben, was eine richtige und gute, aber auch eine falsche und schlechte Ernährung auszeichnet. Gesündere und nachhaltigere Ernährungspraktiken 


\section{Ernährungs- und Verbraucherkommunikation}

scheitern häufig nicht an ausreichenden Informationen oder am Wissen über Alternativen, es ist vielmehr ein Handeln wider besseres Wissen zu beobachten. Die Probleme sind darum eher in einer mangelnden Alltagskompatibilität und -tauglichkeit der Konzepte und Ansätze zu suchen (Rückert-John, 2014).

\section{Essen und Ernährung als Alltagspraktiken und ihr Wandel}

Die bisherigen Betrachtungen werfen die Frage nach alternativen Ansätzen und Betrachtungsweisen der Ernährungs- und Verbraucherkommunikation und -beratung auf. Im Folgenden wird deshalb dafür plädiert, die Perspektive des Defizits auf eine Perspektive der Potenzialerkennung in der Ernährung umzustellen. Damit rücken Essen und Ernährung als Alltagspraktiken und ihr Wandel in den Mittelpunkt der Betrachtung.

Praktiken sind mehr als bloße Handlungen. Sie beruhen auf routiniert befolgten Skripten, die im Lebensverlauf vor allem durch Sozialisation angeeignet werden. Indem sich bestimmte Handlungsabläufe wiederholt mit hoher Frequenz gegenüber gleich erscheinenden Problemlagen als situativ relevant und in ihrer Begründung als gültig erweisen, werden die darin enthaltenen Wissenskomplexe „monothetisch“ komprimiert (Knoblauch, 2005, S. 102 f.), das heißt, die Handlungsphasen werden nicht mehr einzeln reflektiert, sondern im Ganzen automatisch vollzogen (z. B. Einkaufen). Die Entscheidungen, bestimmte Motive und Werte in Handlungen zu verfolgen, werden dadurch implizit. Das heißt nach Polanyi (1985, S. 18 f.), die jeweiligen situativen Umstände, nicht aber die eigentlichen Entscheidungen werden dann als Ursache der Praktiken verstanden, wodurch sie der Reflexion im Normalfall entzogen sind (Reckwitz, 2003, S. 293). Indem Praktiken an kollektiv geltende Wertsetzungen orientiert sind, leiten sie konkrete Handlungen an (Schatzki, 2002). Auf diese Weise können sie individuelle Handlungsfähigkeit und -sicherheit gewährleisten (Shove, 2003, Reckwitz, 2003). Praktiken sind Skripte mit habituellem Charakter, die in der körperlichen Performanz einerseits auf das Individuum, wegen der Wertegrundlage aber andererseits auf die soziale Verfasstheit in Kollektivbezügen und letztlich Gesellschaft verweisen. Indem Alltagspraktiken immer nur problemlos als solche funktionieren können und sie auf dieser Weise der Reflexion unzugänglich sind, können sie bewusst nicht verändert werden, denn „,der Alltag ist im Alltag kein Thema“" (John, 2012, S. 108). Erst wenn die Handlungsskripte, die verketteten Routinen nicht mehr funktionieren und darum als Praktiken scheitern, können sie reflektiert werden. Denn im Scheitern lösen sich die verketteten und monothetisch verdichten Skripte auf. Unter günstigen Umständen und mühsam, auf jeden Fall zeitraubend, können die Gründe erinnert werden, die zu den nun unpraktischen Handlungsvollzügen führten und wieso diese offensichtlich nicht mehr gelten. 


\section{Ernährungs- und Verbraucherkommunikation}

Gewahr wird man dabei, dass Praktiken Strukturen im Sinne von Arrangements (Schatzki, 2010) zum Anlass ihres Vollzuges nehmen und diese damit reproduzieren. So brachte beispielsweise die verstärkte Nachfrage von Bio-Lebensmitteln in Folge der BSE-Krise entsprechende Bio-Supermärkte hervor, die heute in deutschen Großstädten nicht mehr wegzudenken sind, was jedoch den Einkauf von BioLebensmitteln enorm erleichtert. Sachliche Aspekte der Struktur, Materialien, konkrete Räume, aber eben auch Körper ermöglichen den Praktiken ihre eigentümlichen Formen (Schmidt, 2012, S. 55 ff.). Praktiken sind zeitlich doppelt markiert: Einerseits sind sie im ständigen Werden und Vergehen begriffen, doch erweist sich gerade dadurch andererseits ihre Dauerhaftigkeit. In diesem Sinne hängen die ernährungsrelevanten Praktiken der Bedürfnisbefriedigung von bestimmten Arrangements ab. Zugleich sind die Arrangements in ihrer kollektiven Vermittlung aber auch Grundlage der zu Bedürfnissen geformten Motive und Erwartungen. Praktiken müssen sich darum hinsichtlich der Intentionen und den Bedingungen ihrer Erfüllung als adäquat erweisen, um zwischen ihnen zu vermitteln.

Indem Praktiken der Reflexion entzogen sind, funktionieren sie gegenüber unvermeidlichen Variationen der passenden Arrangements. So gesehen sind Praktiken hyperstabil. Gleichwohl können Praktiken scheitern. Schon die Interpretationsbedürftigkeit der vielfältigen, komplexen Umwelt kann Veränderungen von Praktiken veranlassen (Reckwitz, 2003, S. 294). Wenn sich Elemente und Reproduktionsbedingungen des Arrangements der Praktiken ändern (Shove et al., 2012, S. 119 ff.), geraten Praktiken aufgrund ihres Versagens in eine Krisen (z. B. veränderte Einkaufsinfrastrukturen nach Umzug in eine andere Stadt). Solche Krisen können zu inkrementellen Veränderungsanforderungen führen, die sich durch den Austausch von Elementen des monothetischen Wissenskonglomerats bewältigen lassen. Jedoch können Praktiken sich auch als völlig überholt erweisen. Ein strukturell weitreichender Effekt ergibt sich daraus aber noch nicht. Denn im ersten Fall wird der Vollzug der Praktik variiert fortgesetzt, im anderen Fall wird sie einfach irrelevant für die Reproduktion der bereits veränderten Umweltarrangements. Wandeln sich Praktiken aber in einer Weise, die die Reproduktion der Umweltarrangements verändert, erscheinen sie innovativ. Dann haben die Praktiken mithin Ernährung und Konsum weitreichende strukturelle Effekte. Struktureller Wandel heißt dabei, die bisher mehrheitlich praktizierten und für selbstverständlich angesehenen Routinen sowie die hier zugrundeliegenden Wertsetzungen zu verändern und die Transformation zu neuen an Gesundheit und Nachhaltigkeit orientierten Wertsetzungen und Routinen zu unterstützen (Spaargaren \& Van Vliet, 2000).

Veränderungen von Ernährungspraktiken können nur durch ihre nachhaltige Störung, Irritation oder Überraschung angeregt werden. Werden die Praktiken auf diese Weise problematisch, sind sie als mögliche Handlungsvollzüge sichtbar, die nach Alternativen verlangen. Anlässe oder Gelegenheiten für eine solche problematisierende Auflösung von Praktiken zu nicht mehr möglichen Handlungsvollzügen kön- 


\section{Ernährungs- und Verbraucherkommunikation}

nen zum Beispiel biografische Ereignisse sein, wie die Geburt eines Kindes, Krankheit oder Umzug, ergreifende Erlebnisse (wie z. B. Umweltkatastrophen, Filme), aber auch Informationen und Wissen (z. B. gesundheitliche Folgewirkungen fleischlastiger Ernährungsstile) (Schäfer \& Jaeger-Erben 2011, Brunner, 2007, Herde, 2007, Sehrer, 2004). Die Anlässe müssen ein hinreichendes, das heißt zeitlich, sozial und sachlich nachdrückliches Störpotenzial haben, um die individuellen Ernährungspraktiken als Handlungen für Reflexionen zugängig zu machen. Auf dieser Weise werden sie explizit hinsichtlich ihrer Gründe als spezifische Lösungen für Probleme thematisierbar und bewertbar. Nur so können sie einer Veränderung zugeführt werden oder - wenn dies nicht der Fall ist - sich erneut im neuen, durch die Störungen umstrukturierten Arrangement in ihrer Alltagstauglichkeit bestätigen.

Mit der Forschungsperspektive auf soziale Praktiken, kann zunächst ein notwendiges Verständnis über den Ernährungsalltag von Konsumentinnen und Konsumenten gewonnen werden. Damit erfolgt - mit Blick auf die These der Unterschätzung des Ernährungsverhaltens - eine grundsätzliche Anerkennung des Funktionierens, d. h. der Funktionsweise und Rationalität, der alltäglichen Ernährungspraktiken. Zudem kann - vor dem Hintergrund der These der Überschätzung - gezeigt werden, dass bei der Alltagsgestaltung andere Prämissen neben an den Gesundheits- und Nachhaltigkeitsnormen ausgerichtete Informationen und Wissen relevant sind. Klar wird dann: Gesundheit und Nachhaltigkeit kann nicht anstelle solcher Werte treten, sondern muss als Möglichkeit zur besseren Erfüllung der eigentlich im Alltag angestrebten Ziele darstellbar werden.

Zudem macht die Perspektive auf soziale Praktiken deutlich, dass eine breite Sensibilisierung und Aktivierung der Konsumentinnen und Konsumenten auf das Zusammenspiel von einerseits neu zu verändernden Rahmenbedingungen als verhältnispräventive Maßnahmen und andererseits spezifischen verhaltenspräventiven Impulsen angewiesen ist, um alltagsrelevante Selbstverständlichkeiten und routinierte Praktiken aufzubrechen sowie einen entsprechenden Wertewandel in Bezug auf die praktizierten Konsumpräferenzen in Gang zu setzen. Die Verantwortung für eine gesunde Ernährung und einen nachhaltigeren Konsum kann nicht allein den Konsumentinnen und Konsumenten aufgebürdet werden. Es geht vielmehr um eine ,geteilte Verantwortung" (Belz \& Bilharz 2007) und um die Bereitstellung von Strukturen, die als Arrangements viel stärker als bisher gesunde und nachhaltige Ernährungspraktiken überhaupt erst ermöglichen.

\section{Soziale Innovationen im Bereich der Ernährung}

Vor diesem Hintergrund werden seit geraumer Zeit verschiedene neue Organisationsformen, Dienstleistungen und Angebote, wie ,Collaborative Consumption' und Sharing Economy, Commoning, und Gemeingüter-Ökonomie, Prosuming, Upcycling und ReUse diskutiert, die die Konsumgewohnheiten hin zu mehr Nachhaltigkeit 


\section{Ernährungs- und Verbraucherkommunikation}

verändern können (Rückert-John et al., 2014a, Heinrichs \& Grunenberg, 2012). Auch im Bereich der Ernährung gibt es zahlreiche Initiativen und Projekte, die alternative Praktiken der Ernährung ermöglichen. Hierzu zählen unter anderem ErzeugerVerbraucher-Gemeinschaften, Projekte des Urban Gardening, Food-SharingInitiativen oder Containern, mietbare Gemüsegärten, das Tafeln, Initiativen wie „Mundraub“, „Land Share“, Community Supported Agriculture, interkulturelle Gärten, die Regionalwert AG oder das „Guerilla Gardening“. Solche Angebote und Initiativen werden auch als ,soziale Innovationen“ bezeichnen (Rückert-John, 2015). Im Unterschied zum gängigen Innovationsverständnis, das vor allem auf die ökonomische Verwertung technologische Artefakte zielt, wird davon ausgegangen, dass durch sogenannte soziale Innovationen allägliche Praktiken direkt angesprochen werden. Die Initiativen und Projekten entwickeln nämlich aus dem Alltag heraus neue Lösungen für wahrgenommene, gegenwärtige Probleme von Praktiken. Die Suche nach Möglichkeiten einer gesunden und nachhaltigen Ernährung erfolgt entlang der Potenziale im Alltag und nicht (!) mit Blick auf ernährungsphysiologische oder umweltrelevante Defizite bestimmter Ernährungsweisen. Wahrgenommene Defizite können lediglich als Störungen fungieren und zur Reflexion über die Praktiken führen; sie bieten jedoch keinerlei Orientierung für tatsächlich mögliche Alternativen im Alltag. Bislang finden diese Angebote für alterative Praktiken jedoch eher in Nischen bei den sogenannten „Pionieren des Wandels“ Resonanz und Akzeptanz. Prospektiv muss sich erst noch beweisen, ob diese Ideen in der Gesellschaft Verbreitung finden und sich damit dauerhaft stabilisieren. Erst wenn sie sich als selbstverständliche Praktiken in der Gesellschaft verbreiten, lassen sich die Initiativen und Projekte als Innovationen bezeichnen. ten, die Regionalwert AG oder das „Guerilla Gardening“. Solche Angebote und Initiativen werden auch als, soziale Innovationen“ bezeichnen (Rückert-John, 2015). Im Unterschied zum gängigen Innovationsverständnis, das vor allem auf die ökonomische Verwertung technologische Artefakte zielt, wird davon ausgegangen, dass durch sogenannte soziale Innovationen alltägliche Praktiken direkt angesprochen werden. Die Initiativen und Projekten entwickeln nämlich aus dem Alltag heraus neue Lösungen für wahrgenommene, gegenwärtige Probleme von Praktiken. Die Suche nach Möglichkeiten einer gesunden und nachhaltigen Ernährung erfolgt entlang der Potenziale im Alltag und nicht (!) mit Blick auf ernährungsphysiologische oder umweltrelevante Defizite bestimmter Ernährungsweisen. Wahrgenommene Defizite können lediglich als Störungen fungieren und zur Reflexion über die Praktiken führen; sie bieten jedoch keinerlei Orientierung für tatsächlich mögliche Alternativen im Alltag. Bislang finden diese Angebote für alterative Praktiken jedoch eher in Nischen bei den sogenannten „Pionieren des Wandels" Resonanz und Akzeptanz. Prospektiv muss sich erst noch beweisen, ob diese Ideen in der Gesellschaft Verbreitung finden und sich damit dauerhaft stabilisieren. Erst wenn sie sich als selbstverständliche Praktiken in der Gesellschaft verbreiten, lassen sich die Initiativen und Projekte als Innovationen bezeichnen. 


\section{Ernährungs- und Verbraucherkommunikation}

\section{Was heißt das für die Ernährungs- und Verbraucherberatung und -kommunikation?}

Die Frage, die sich abschließend stellt, ist die nach den Schlussfolgerungen aus einer praxistheoretischen Perspektive für die Ernährungs- und Verbraucherberatung sowie -kommunikation. Zum einen wird mit ihr eine konsequente Umstellung der Betrachtungsperspektive - vom Defizit zum Potenzial - angeraten. Hierbei sollte nicht länger die Kluft zwischen Bewusstsein oder Wissen und dem Verhalten beklagt werden. Vielmehr geht es darum, beim Alltag und den Ernährungspraktiken anzusetzen und auf das dabei zugrunde gelegte Wissen und Können zu schließen. Ziel sollte es hierbei sein, den Ess- und Ernährungsalltag, der auf routinierten, habitualisierten und vorreflexiven Praktiken gründet, zunächst zu ergründen und in diesem Sinne dessen Rationalität zu ,entdecken'. Hierbei geht es darum, die vorherrschenden Alltagslogiken anzuerkennen, um so von ihnen zu lernen, statt sie mittels moralischer Ernährungskommunikation zu diskreditieren. Nur so können die strukturellen Bedingungen des Ess- und Ernährungsalltags verstanden und Veränderungen angeregt werden. Letztlich geht es darum, explizite Anlässe als Gelegenheitsfenster für Veränderungen zu erkennen und gegebenenfalls zu verstärken (z. B. die Geburt eines Kindes und die damit häufig verbundene Gründung einer Familie). Diese so verstandenen Interventionen werden nur eine Chance auf Erfolg haben, wenn sie milieu- und lebensphasenspezifische Besonderheiten berücksichtigen. Doch ist dies nur der erste Schritt.

Aus Sicht der Praxisforschung (Spurling et al., 2013) wird ein weiter reichendes Re-Framing konventioneller Alltagspraktiken, so auch von Ernährungspraktiken empfohlen. Hierunter lässt sich - mit Blick auf Ernährung - ein Austausch problematischer Elemente von Praktiken verstehen (1). So können zum Beispiel Sinngehalte einzelner Produkte, wie Bio-Lebensmittel, kommunikative umgedeutet werden. Dann würden Bio-Lebensmittel als nachhaltige Produktalternative nicht diejenigen Produkte angesehen werden, die zu teuer sind, sondern Produkte aus nichtnachhaltiger Produktion, die zu billig sind und keinen adäquaten Preis abbilden. Des Weiteren empfiehlt sich die Strategie, nicht nur Elemente, sondern komplette Ernährungspraktiken durch andere zu ersetzen (2). So könnten beispielsweise schulische Versorgungsstrukturen dahingehend umstrukturiert werden, dass Getränkeautomaten und die als ungesund geltenden Lebensmittel konsequent aus der Schule ,verbannt ${ }^{*}$ werden und stattdessen ,gesunde“ Alternativen angeboten werden. Da Ernährungspraktiken immer auch auf andere Praktiken, zum Beispiel im Schulalltag, verweisen, müssen diese wechselseitigen Beziehungen in ihrer sachlichen und zeitlichen Abfolge ,unterbrochen' werden (3), wenn Veränderungen von Ernährungspraktiken längerfristig von Erfolg sein sollen. So konkurriert die Zielsetzung einer gesunden und nachhaltigen Ernährung im schulischen Alltag immer auch mit anderen Ansprüchen, aus denen Notwendigkeiten und Verpflichtungen abgeleitet werden. Die drei genannten Strategien können zwar einzeln verfolgt werden, sie lassen sich aus einer 


\section{Ernährungs- und Verbraucherkommunikation}

übergreifenden Perspektive aber auch miteinander kombinieren, wenn zum Beispiel ein neues und integriertes schulisches Verpflegungskonzept entwickelt wird, wie dies etwa von den entsprechenden Vernetzungsstellen für Schulverpflegung in den Bundesländern angestrebt wird.

Mit den genannten Empfehlungen wird nicht der Anspruch auf Vollständigkeit erhoben. Sie machen aber deutlich, dass der als notwendig erachtete Wandel von Essund Ernährungspraktiken in der modernen Gesellschaft maßgeblich nur dadurch befördert werden kann, wenn nicht einseitig auf Verhaltensprävention gesetzt wird, sondern vor allem auch Ermöglichungsstrukturen für eine gesunde und nachhaltigere Ernährung geschaffen werden.

\section{Literatur}

Belz, F.-M. \& Bilharz, M. (2007). Nachhaltiger Konsum, geteilte Verantwortung und Verbraucherpolitik: Grundlagen. In F.-M. Belz, G. Karg, Georg \& D. Witt (Hrsg.), Nachhaltiger Konsum und Verbraucherpolitik im 21. Jahrhundert. Metropolis Verlag.

BMUB (Bundesumweltministerium) und UBA (Umweltbundesamt) 2013. Umweltbewusstsein in Deutschland 2012. Dessau: Umweltbundesamt.

[www.umweltbundesamt.de/sites/default/files/medien/publikation/long/4396.pd].

Brand, K.-W. (2011). Umweltsoziologie und praxistheoretischer Zugang. In: M. Groß (Hrsg.), Handbuch Umweltsoziologie (S. 173-198). Wiesbaden: VS Verlag.

Brunner, K.-M. (2007). Ernährungsalltag im Wandel. Chancen für Nachhaltigkeit. Wien: Springer Verlag.

Busse, T. (2006). Die Einkaufsrevolution. Konsumenten entdecken ihre Macht. Blessing Verlag.

Eberle, U., Hayn, D., Rehaag, R. \& Simshäuser, U. (2006). Ernährungswende. Eine Herausforderung für Politik, Unternehmen und Gesellschaft. München: Oekom.

Heinrichs, H. \& Grunenberg, H. (2012). Sharing Economy - Auf dem Weg in eine neue Konsumkultur? Lüneburg: Centre for Sustainability Management.

Herde, A. (2007). Nachhaltige Ernährung im Übergang zur Elternschaft. Berlin: Mensch \& Buch.

Hirschfelder, G. (2005). Europäische Esskultur. Eine Geschichte der Ernährung von der Steinzeit bis heute. Campus Verlag.

John, R. (2012). Innovativität der Alltagsroutinen - Stabilität, Veränderung und Umweltaffinität. Beiträge zur Sozialinnovation, Nr. 8. Berlin: Institut für Sozialinnovation.

John, R., Rückert-John, J. \& Bormann, I. (2014). Repräsentativumfrage zum Umweltbewusstsein und Umweltverhalten im Jahr 2012 einschließlich sozialwissenschaftlicher Analysen (Projektabschlussbericht). 


\section{Ernährungs- und Verbraucherkommunikation |}

[www.umweltbundesamt.de/sites/default/files/medien/378/publikationen/texte_7 8_2014_repraesentativumfrage.pdf].

Klotter, C. (2014). Das Scheitern der Diäten. In R. John \& A. Langhof, A, (Hrsg.), Scheitern - Ein Desiderat der Moderne? (S. 47-60). Wiesbaden: VS Springer,.

Knoblauch, H. (2005). Kulturkörper. In M. Schoer (Hrsg.) Soziologie des Körpers (S. 92-113).Frankfurt am Main: Suhrkamp.

Max Rubner Institut (Bundesforschungsinstitut für Ernährung und Lebensmittel) (Hrsg.). (2008). Ergebnisbericht, Teil 1, Nationale Verzehrsstudie II. Karlsruhe.

Pfeiffer, S. (2014). Die verdrängte Realität: Ernährungsarmut in Deutschland: Hunger in der Überflussgesellschaft. Essentials. Springer VS.

Polanyi, M. (1985). Implizites Wissen. Frankfurt (Main): Suhrkamp.

Reckwitz, A. (2003). Grundelemente einer Theorie sozialer Praktiken. Zeitschrift für Soziologie, 32(4), 282-301.

Reisch, L. \& Gwozdz, W. (2011). Von der „Macht der Defaults“ und vom „sanften Stupsen": Verhaltensökonomische Erkenntnisse als Impulse für eine effektive Ernährungspolitik. In A. Ploeger, G. Hirschfelder, Gunther \& G. Schönberger (Hrsg.), Die Zukunft auf dem Tisch. Analysen, Trends und Perspektiven der Ernährung von morgen (S. 323-336). Wiesbaden: VS Springer.

Rückert-John, J. (2014). Reflexionen über den Ernährungsalltag Jugendlicher. In H.W. Hoefert \& C. Klotter (Hrsg.), Krankheitsprävention in der Kontroverse (S. 91-110). Lengerich: Pabst Science Publishers.

Rückert-John, J. (2015). Nachhaltiger Konsum durch soziale Innovationen. Kulinaristik. Wissenschaft-Kultur-Praxis.

Rückert-John, J. (2015). Meat consumption and Sustainability - How is a change of consumer behavior possible? In H. K. Biesalski, A. Drewnowski, J. Dwyer, Johanna T. \& S. Strain (Hrsg.), Nutrition Security in a Changing World: Impact of Global Sociodemographic and Socioeconomic Changes on Nutrition. Springer (im Erscheinen).

Schäfer, M. \& Jaeger-Erben, M. (2011). Lebensereignisse als Gelegenheitsfenster für nachhaltigen Konsum? Die Veränderung alltäglicher Lebensführung in Umbruchsituationen. In R. Defila et al. (2011), Wesen und Wege nachhaltigen Konsums. München: Ökom Verlag.

Rückert-John, J., Jaeger-Erben, M. \& Schäfer, M. (2014a). Soziale Innovationen im Aufwind. Ein Leitfaden zur Förderung sozialer Innovationen nachhaltigen Konsum. Hrsg. Umweltbundesamt.

Schatzki, T. (2002). The Site of the Social: A Philosophical Account of the Constitution of Social Life and Change. Pennsylvania State: University Press.

Schatzki, T. (2010). Materiality and Social Life. Nature and Culture, 5(2), 123-149.

Schmidt, R. (2012). Soziologie der Praktiken. Frankfurt am Main: Suhrkamp.

Sehrer, W. (2004). Krankheit als Chance für nachhaltige Ernährungsumstellungen. [www.konsumwende.de/downloads_fr.htm]. 


\section{Ernährungs- und Verbraucherkommunikation}

Shove, E. (2003). Comfort, Cleanliness and Convenience. The Social Organization of Normality. Oxford: Berg.

Shove, E., Pantzar, M. \& Watson, M. (2012). The Dynamics of Social Practice. Los Angeles: Sage.

Spaargaren, G. \& Vliet, B.J.M. van (2000). Lifestyles, Consumption and the Environment: The Ecological Modernisation of Domestic Consumption. Environmental Politics, 9(1). 50-77.

Spurling, N., McMeekin, A., Shove, E., Southerton, D. \& Welch, D. (2013). Interventions in practice: Reframing policy approaches to consumer behaviour. Sustainable Practices Research Group Report.

[www.sprg.ac.uk/uploads/sprg-report-sept-2013.pdf].

Statisches Bundesamt (2015). Anteil der Ausgaben der privaten Haushalte in Deutschland für Nahrungsmittel, Getränke und Tabakwaren an den Konsumausgaben in den Jahren in den Jahren 1850 bis 2014.

[http://de.statista.com/statistik/daten/studie/75719/umfrage/ausgaben-fuernahrungsmittel-in-deutschland-seit-1900].

Wissenschaftlicher Beirat für Agrarpolitik beim Bundesministerium für Ernährung und Landwirtschaft (2015). Wege zu einer gesellschaftlich akzeptierten Nutztierhaltung.

[www.bmel.de/SharedDocs/Downloads/Ministerium/Beiraete/Agrarpolitik/Guta chtenNutztierhaltung.pdf?_blob=publicationFile].

\section{Verfasserin}

Prof. ${ }^{\text {in }}$ Dr. Jana Rückert-John

Hochschule Fulda

Fachbereich Oecotrophologie

Professur für „Soziologie des Essens“

Leipziger Str. 123

D-36037 Fulda

E-Mail: jana.rueckert-john@he.hs-fulda.de 